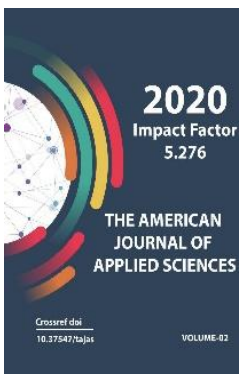

Copyright: Original content from this work may be used under the terms of the creative commons attributes 4.0 licence.

\section{Degradation Features Of Polyethylene And Gelatin Compositions}

\author{
N.R.Ashurov \\ Institute Of Chemistry And Physics Of Polymers Of The Academy Of Sciences Of The Republic \\ Of Uzbekistan, Tashkent, Uzbekistan
}

\section{Sh.G.Sadikov}

Institute Of Chemistry And Physics Of Polymers Of The Academy Of Sciences Of The Republic Of Uzbekistan, Tashkent, Uzbekistan

\section{N.F.Normurodov}

Institute Of Chemistry And Physics Of Polymers Of The Academy Of Sciences Of The Republic Of Uzbekistan, Tashkent, Uzbekistan

\section{Q. N.Berdinazarov}

Institute Of Chemistry And Physics Of Polymers Of The Academy Of Sciences Of The Republic Of Uzbekistan, Tashkent, Uzbekistan

\section{E.O.Khakberdiev}

Institute Of Chemistry And Physics Of Polymers Of The Academy Of Sciences Of The Republic Of Uzbekistan, Tashkent, Uzbekistan

\title{
ABSTRACT
}

In this work, biodegradable properties of gelatin and polyethylene compositions, in addition to, influence of ultra violet radiation and peroxide groups were studied. In order to obtain thermoplastic gelatin water and glycerin were used as a plasticizer. For polyethylene/gelatin blends, to provide compatibility polyethylene functionalized with maleic anhydride. It was found that as gelatin content increases, biodegradation also grows while mechanical properties (elastic module and yield strength) decrease.

\section{KEYWORDS}

Biodegradation, functionalization, gelatin, glycerin, polyethylene, polymer compositions.

\section{INTRODUCTION}

The packaging industry is in dire need of efficient biodegradable polymer systems. In this aspect, it is of interest to create compositions containing, in addition to the high-molecular basis, organic fillers such as starch, cellulose, amylose, amylopectin, dextrin, et cetera which are a nutrient surrounding for microorganisms [1-5]. These 
compositions should also be characterized by the provided mechanisms of physicochemical and biological transformations under the influence of environmental factors.

The most technologically advanced method for producing polymer-filler composites is the direct mixing of the components in the polymer melt. In this case, the filler is present in the polymer matrix in the form of agglomerates $10-100 \mu \mathrm{m}$ in size [6-8]. The particle size of the dispersed phase in the process of mixing the components depends on the ratio of the viscosities of the mixed components, interfacial tension, and the magnitude of the applied shear stress. Due to the coalescence of dispersed phase particles at the stage of completion, after stress relief, a coarsely dispersed morphology with a particle size of more than $100 \mu \mathrm{m}$ is recorded. The morphology of the polymer mixture, in particular, the polyethylene/gelatin system under consideration, is easily controlled by the introduction or their creation (compatibilizers) in the form of grafted or block copolymers in the process of mixing the components. With this approach, the desired particle size in the range of hundreds of nanometers can be achieved and stabilized.

Furthermore, such compositions, due to loosening (amorphization) of the crystallization of polyethylene matrix and facilitating the access of microorganisms to gelatin, have higher biodegradable characteristics.

The destruction of polymeric macromolecules can proceed in two ways: physicochemical, by hydrolysis under the action of acidic or alkaline environment, or under the action of bacterial and fungal cultures, which carry out the enzymatic degradation of polymers. The rates of biodegradation by hydrolysis are generally lower than those caused by microorganisms. There are no enzymes capable of degrading synthetic polymers such as polyolefins or polyvinyl polymers. However, it is precisely these classes of polymers that make up the majority of household waste in all industrialized countries [9].

In works $[10,11]$, the authors propose a variant of modification of PE with maleic anhydride (MA) in order to increase its compatibility with starch to obtain biodegradable materials for packaging. The resulting product can enter into chemical interaction with starch and act as an acylating agent for starch hydroxo groups, which ultimately made it possible to achieve high biodegradable properties.

Following this approach, in this work, we studied the features of biodegradation of compositions based on maleinized polyethylene (PEMA) and gelatin.

\section{RESEARCH METHODS AND MATERIALS}

We used in the work: LLDPE grade F-0320, $d=$ $0.920 \mathrm{~g} / \mathrm{cm}^{3}, \mathrm{MFI}=2.5 \mathrm{~g} / 10 \mathrm{~min}$ (at a load of 2.16 kgf). Manufacturer - Shurtan Gas Chemical Complex of Republic of Uzbekistan; Food gelatin grade P - 200 (GOST 11293 - 2019). Manufacturer OJSC "MOZHELIT", Belarus; Maleic anhydride $\mathrm{C}_{4} \mathrm{H}_{2} \mathrm{O}_{3}$, analytical grade, colorless rhombic crystals, $M_{r}=98,06 \mathrm{~g} / \mathrm{mol}$, was distilled at $\mathrm{T}_{\mathrm{m}}=84^{\circ} \mathrm{C} / 14 \mathrm{~mm} \mathrm{Hg}, \mathrm{Tm}=$ $60^{\circ} \mathrm{C}, \rho^{60}=1.3140 \mathrm{~g} / \mathrm{cm}^{3}$.

LLDPE functionalization with maleic anhydride (LLDPE-g-MA) was carried out on a Brabender plastograph (Plasticorder Brabender OHGDUISBURG Germany), with a cam speed of $98 \mathrm{rpm}$ and at a temperature of $180 \pm 5^{\circ} \mathrm{C}[8]$. The concentration of the grafted maleic anhydride was varied in the range of 0.5-5.0 wt\%.

Polymer blends based on LLDPE-g-MA and gelatin were obtained on a Brabender plastograph, for $30 \mathrm{~min}$, at $50 \mathrm{rpm}$ and $180 \pm 5$ ${ }^{\circ} \mathrm{C}$ by adding plasticized gelatin to the LLDPEg-MA melt. 
To determine the water absorption, the samples of polymer mixtures in the form of films were completely immersed in a static water bath at $25^{\circ} \mathrm{C}$. The samples were carefully wiped to remove surface water before weighing. The increase in the mass of the wet sample determines the degree of water absorption. The amount of absorbed water was monitored after 24 and 48 hours. Water uptake was determined using the following equation:

\section{$\mathbf{W g}=($ Wa-Wo $) /$ Wo $\times 100$}

where $\mathrm{W}_{\mathrm{g}}$ is the water absorption percentage of obtained samples as a result of water, $\mathrm{W}_{\mathrm{a}}$ is the mass of the sample after immersion in water, and $W_{0}$ is the mass of the sample before immersion in water.

The biodegradation study of polymer compositions was carried out in the form of films in specially prepared soil (with a moisture content of at least $25 \%$ ). During the test period, the soil moisture was maintained at 30\% and the samples were immersed to a depth of $15 \mathrm{~cm}$. The embedded samples in the soil were removed every 10 days of biodegradation tests, washed in ethanol, wiped off, and equilibrated in a desiccator until getting constant weight before being recorded mass. The weight loss of the sample (W) in \% was determined depending on the number of days as follows:

$$
\mathbf{W}=\left(\mathrm{W}_{\mathrm{0}}-\mathrm{W}_{\mathrm{i}}\right) / \mathrm{Wo} \times 100
$$

$(\Delta \mathrm{W}):$

Weight loss in\% after every 10 days

$$
\Delta \mathrm{W}=\left(\mathrm{W}_{\mathrm{i}}-\mathrm{W}_{\mathrm{i}-1)} / \mathrm{W}_{\mathrm{i}} \times 100\right.
$$

where $\mathbf{W}_{\mathbf{i}}$ is the final mass every 10 days, $\mathbf{W}_{\mathrm{i}-1}$ - initial mass every 10 days.
Physical and mechanical tests of the samples were carried out on a universal testing machine Instron 3366 (USA) in the uniaxial tension mode at a speed of $20 \mathrm{~mm} / \mathrm{min}$, in accordance with the requirements established by ASTM D638-99 "Standard test method for the strength properties of plastics".

For the analysis, at least five samples of the test material were used, made in the form of double-sided blades with a thickness of $2 \mathrm{~mm}$.

\section{RESULTS AND ITS DISCUSSION}

Biodegradation studies. Biodegradation is the chemical decomposition of materials caused by living organisms (bacteria, fungi, etc.) or by the action of enzymes. The main attacking agents in biodegradation are microorganisms such as actinomycetes, fungi, and bacteria. The polymer blend samples are composed of an LLDPE-gelatin graft copolymer and free gelatin. Gelatin, a polyamide protein, is a biodegradable polymer. When grafting gelatin to LLDPE by functional maleic groups [10], the process of crystallization of the mixed components and the reaction product becomes difficult. In all likelihood, the presence of biodegradable gelatin in the graft copolymer will contribute to a certain extent to the degradation of PE. The biodegradation of various samples, in the soil, is shown in Fig. 1. The figure shows that the weight loss of the samples increases with an increase in the residence time in the soil. This indicates that the samples were continuously degraded over time. The percentage of weight loss was determined by subtracting the weight of the sample taken on a specific day (for example, every 10 days) from the original weight, that is, the weight of the sample at the time of testing. 


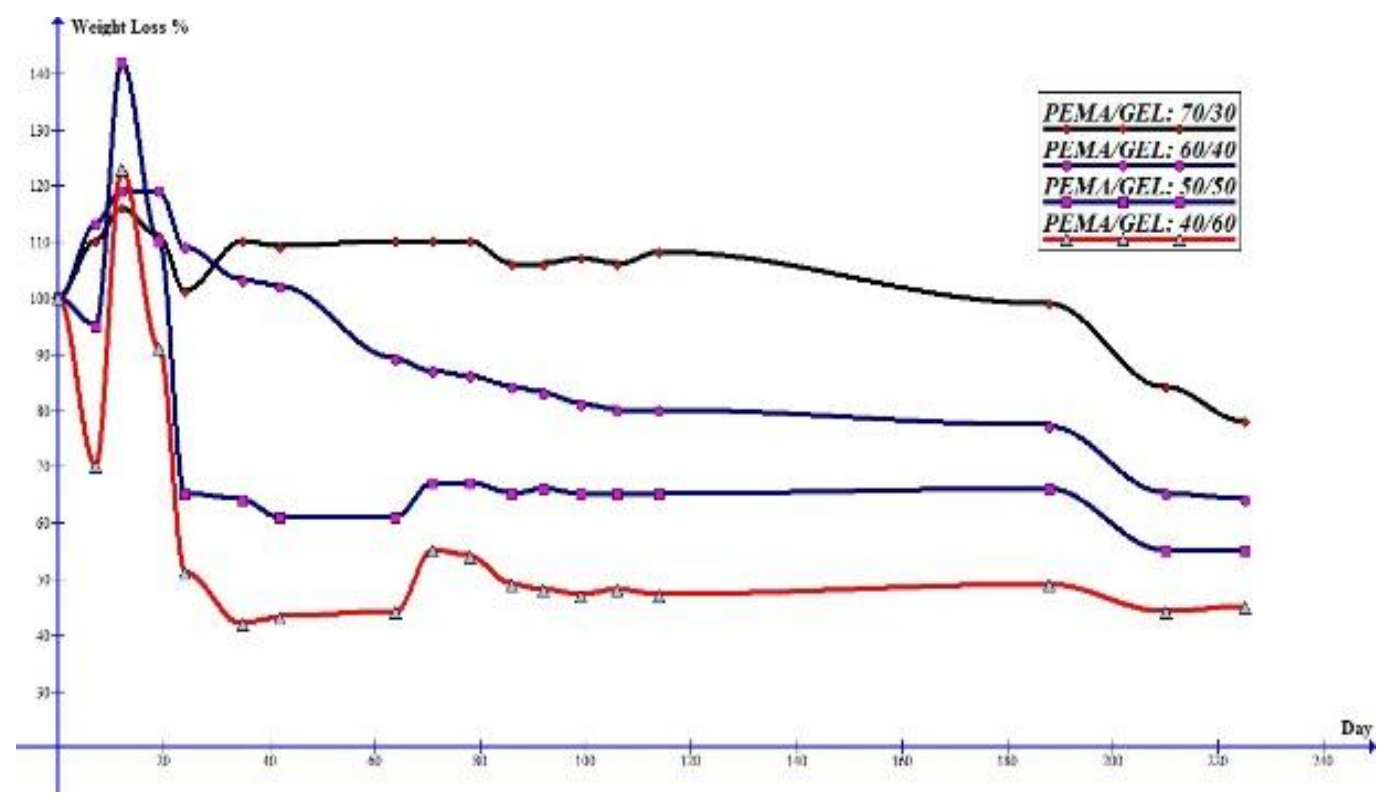

Fig 1. Change in the mass of samples with different PE/gelatin blends by the time of degradation in the soil.

As can be seen from Fig. 1, for all samples, the weight loss of the composition observed over time is proportional to the gelatin concentration and proceeds only through the stage of preliminary swelling of the sample, with the exception of the composition containing $50 \%$ and $60 \%$ by weight gelatin.

When comparing the kinetic curves of biodegradation of the composition, it can be seen that they have two characteristic biodegradation behavior. The common thing is that for all samples the maximum swelling is achieved on the 12th day of being in the soil. Moreover, for gelatin content of 30\% and 40\% compositions, there is a gradual swelling, and for $50 \%$ and $60 \%$, it occurs through the process of decomposition (weight loss), observed within 7 days of being in the soil. As we have shown earlier [10], during the formation of polymer-polymer mixtures, a copolymer is formed due to the reaction between maleic groups grafted to LLDPE and amino groups of gelatin, which, as a rule, binds an excess of unreacted gelatin (hydrogen bonds), leading to an increase in the bulk the proportion of dispersed gelatinous phase [12]. This phenomenon has a negative effect on the strength and modulus of elasticity, accompanied by an increase in the deformability of the obtained samples (Table 2). As can be seen from Table 2, an increase in the concentration of gelatin leads to a drop in tensile strength by $13 \%$ and an increase in elongation at break by $28 \%$. 
Table 1

The effect of gelatin concentration on the physicomechanical properties of the composition.

\begin{tabular}{|c|c|c|c|c|}
\hline \multicolumn{2}{|c|}{ Sample composition } & \multirow{2}{*}{$\begin{array}{c}\text { Yield Strength, } \\
\text { [MPa] }\end{array}$} & \multirow[t]{2}{*}{ Elongation, [\%] } & \multirow{2}{*}{$\begin{array}{l}\text { Young's } \\
\text { module, } \\
{[\mathrm{MPa}]}\end{array}$} \\
\hline $\begin{array}{l}\text { LLDPE-g-MA( MA- } \\
\text { 1,5\% wt.) }\end{array}$ & $\begin{array}{c}\text { Plasticized gelatin } \\
\text { (gelatin/ } \\
\text { glycerin/water - } \\
4 / 1 / 1 \text { ) }\end{array}$ & & & \\
\hline 70 & 30 & $13,48 \pm 1,3$ & $117 \pm 16,12$ & $236 \pm 20,35$ \\
\hline 46 & 54 & $11,73 \pm 0,9$ & $163 \pm 6,4$ & $96 \pm 7,52$ \\
\hline
\end{tabular}

As you know, gelatin undergoes rapid biodegradation when exposed to moisture and microorganisms. Figure 2 shows $\Delta \mathrm{W}$ versus test time. The dependencies shown in Fig. 2 can be used to make decision about relative rates of the processes observed in the samples of the composition during biodegradation in the soil every 10 days.

When comparing Fig. 1 and Fig. 2, it can be seen that for compositions, which contain 30\% and $40 \%$ of gelatin, after 7 days of swelling (Fig. 2), a weight loss is observed, while in Fig. 1 its gain is observed (up to 19 days being in the soil) in relation to the original. From this, it follows that in the indicated composites, in the periods between 7 and 19 days of being in the soil, two processes occur - swelling and degradation of samples, and their swelling rates are the same, and degradation of a $40 \%$ sample occurs faster at the second stage and, as a result, generally throughout the entire period of being in the soil.

Fig. 2 shows that for all samples of the composition there is a tendency for the cyclical processes of swelling-degradation in the entire study interval, that is, each stage of degradation is preceded by swelling of the composite, at which an increase in the mass of the sample is observed. Composites with $50 \%$ and $60 \%$ gelatin content are characterized by high rates of swelling (weight gain) and degradation (weight loss), proceeding in parallel up to 24 days in the soil. Further, the swelling rate for a sample with $50 \%$ filling increases (Fig. 2). 


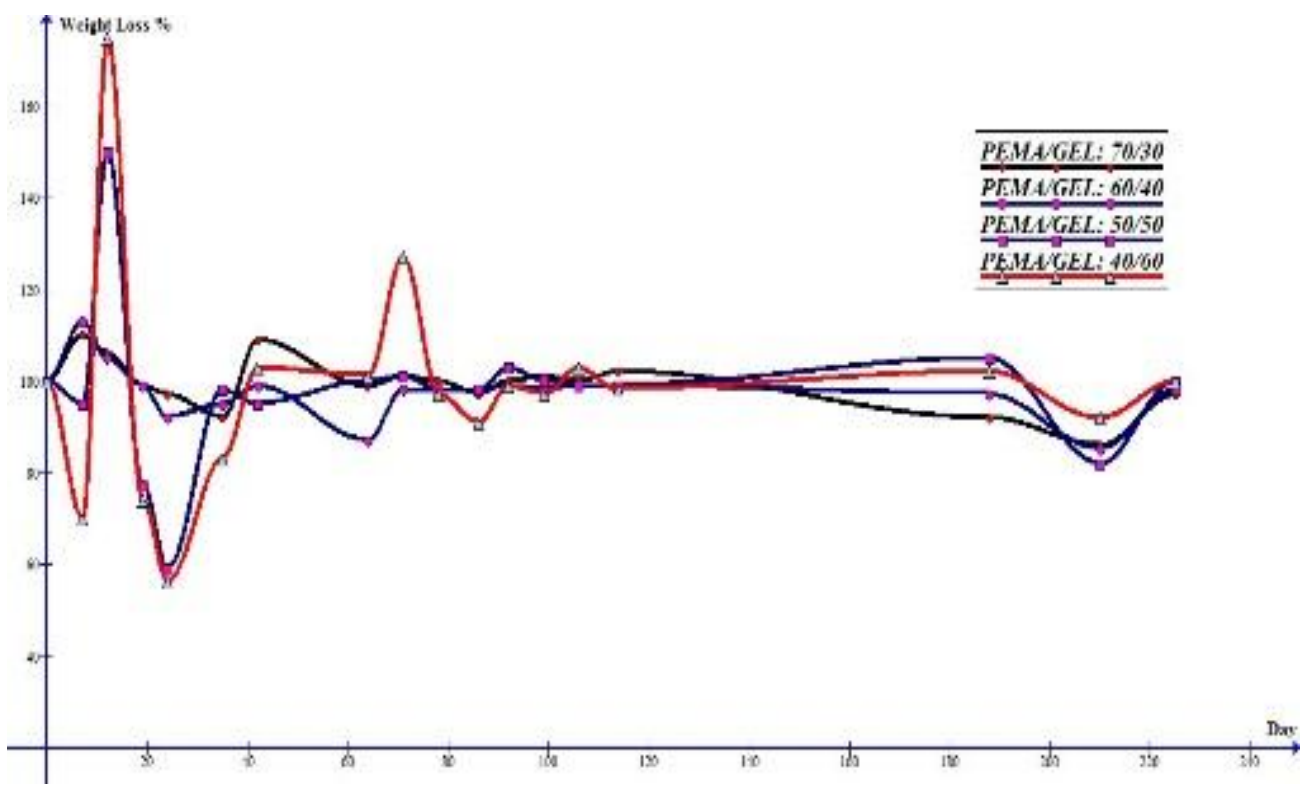

Fig. 2. change in the mass of samples for each successive - 10 days of degradation in the soil,\%.

For composite samples with 50\% and 60\% gelatin content, a distinctive feature is a more pronounced cyclicity of successive processes of swelling and degradation, which is determined by the amount of gelatin, and when it is exhausted (degraded), the rates of these processes are significantly slowed down and practically compared with each other (Fig. 1). ). Thus, these compositions undergo rapid degradation within 7 days at a rate proportional to the concentration of gelatin in the composite (Fig. 2). Further, by the 12th day of being in the soil, the mass of the samples again reaches the maximum, and by the 24 th day, the minimum value, et cetera.
It is known that plastic products after the expiration of their service life are most often buried, where it is exposed to the effects of moisture in the form of precipitation and solar radiation. We tried to simulate these processes, namely, solar irradiation by exposing the obtained composites to UV radiation with a DRT-400 lamp for 10 hours, and the oxidation process by treating the surface of the samples with benzoyl peroxide. Subsequently, the obtained samples were subjected to destruction in the soil; Fig. 3 shows the data obtained using the example of a composition of $40 \%$ gelatin. 


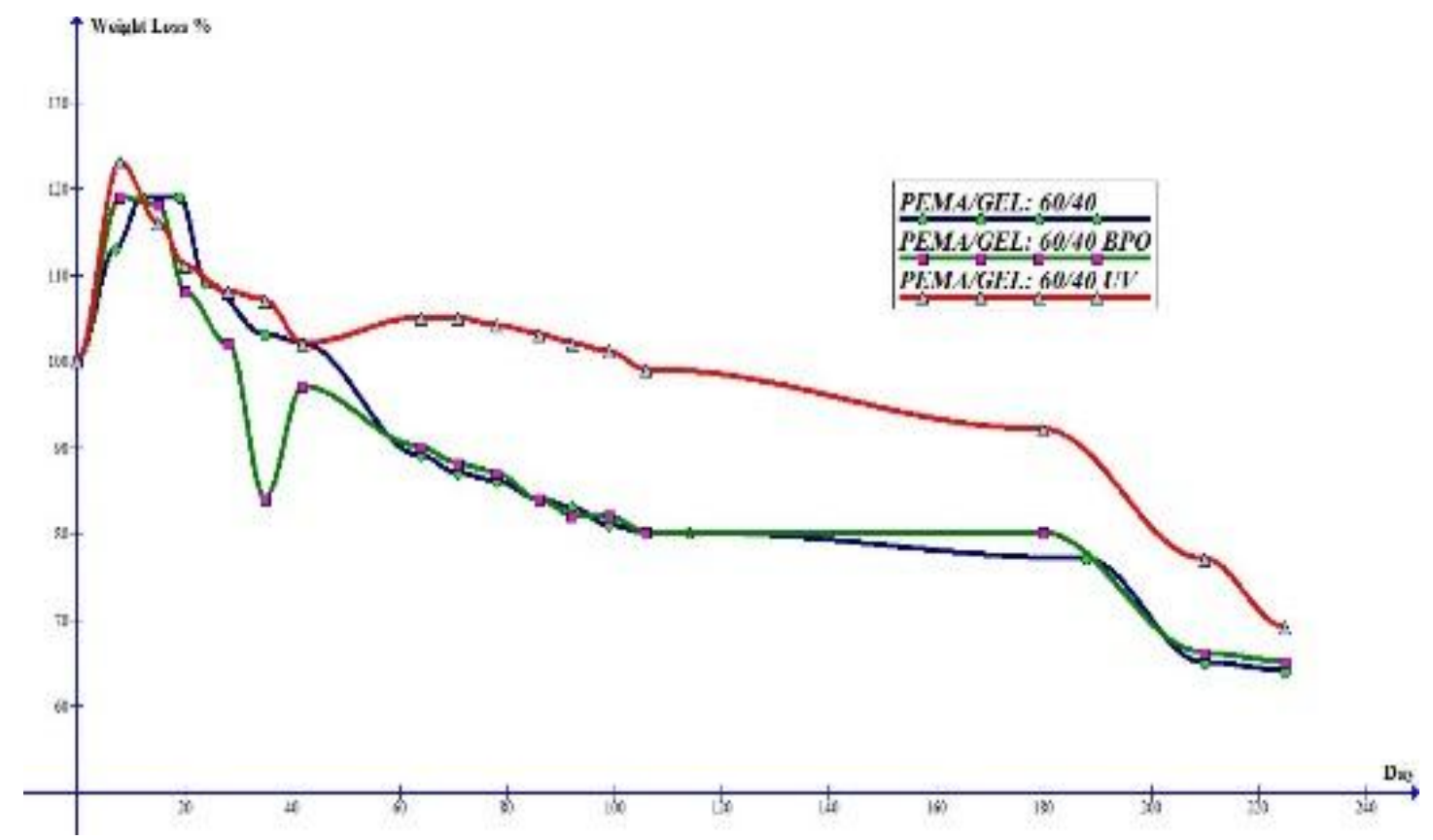

Fig. 3. Influence of UV radiation and peroxide treatment on the degradation of samples in soil.

As can be seen in Fig. 3, the treatment of the sample with UV radiation leads to some of its structural changes, which is confirmed by the preservation of swelling in the soil, as well as a decrease in the rate of the degradation process in comparison with the initial sample.

Peroxide treatment leads to surface oxidation, which contributes to an increase in the rates of swelling and degradation (Fig. 3).

\section{CONCLUSION}

A copolymer of maleated polyethylene with gelatin can bind a certain part of the free gelatin through hydrogen bonds in the form of a separate phase. At the same time, the obtained films with high gelatin content are

more plastic. When carrying out the biodegradation of samples in the soil, a proportionality was revealed between the concentration of gelatin and the rate of decomposition, and after the degradation of the main part of the gelatin, these rates are practically independent of filling. It has been shown that UV treatment promotes some structuring and slowing down, and peroxide treatment increases the rates of swelling and degradation. The discovered phenomenon opens up approaches for the formation of effective biodegradable systems.

\section{REFERENCES}

1. Ge, L., Zhu, M., Li, X., Xu, Y., Ma, X., Shi, R., ... Mu, C. (2018). Development of active rosmarinic acid-gelatin biodegradable films with antioxidant and long-term antibacterial activities. Food Hydrocolloids, 83, 308-316.

2. Rajan, M., \& Raj, V. (2013). Formation and characterization of chitosan-polylacticacidpolyethylene glycol-gelatin nanoparticles: A novel biosystem for controlled drug delivery. Carbohydrate Polymers, 98(1), 951-958. 
3. Alidadi-Shamsabadi, M., Behzad, T., Bagheri, R., \& Nari-Nasrabadi, B. (2014). Preparation and characterization of lowdensity polyethylene/thermoplastic starch composites reinforced by cellulose nanofibers. Polymer Composites, 36(12), 2309-2316.

4. Sarker, B., Dey, K., \& Khan, R. A. (2011). Effect of Incorporation of Polypropylene on the Physico-Mechanical and ThermoMechanical Properties of Gelatin Fiber Based Linear Low Density Polyethylene Bio-foamed Composite. Journal of Thermoplastic Composite Materials, 24(5), 679-694.

5. Akhmetkhanov R.M., Minsker K.S., Zaikov G.E., Plast. masses. 2006. No. 8., pp. 6-9.

6. M. Mizanur Rahman, Rezaul Karim, A. I. Mustafa \& Mubarak A. Khan (2012) Preparation and Characterization of Bioblends from Gelatin and Linear Low Density Polyethylene (LLDPE) by Extrusion Method, Journal of Adhesion Science and Technology, 26:8-9, 1281-1294

7. Khanna, N. D., Kaur, I., Bhalla, T. C., \& Gautam, N. (2010). Effect of biodegradation on thermal and crystalline behavior of polypropylene-gelatin based copolymers. Journal of Applied Polymer Science, n/a-n/a.

8. E.O. Khakberdiev, N.R. Ashurov, Sh.G. Sadykov, N.Sh. Ashurov // in: "Science, Research, Development \#13. TechnicsandTechnology ", Berlin. 3031.01.2019.P.30-32.

9. Environmental Protection Agency. Plastics.

EPA

https://www3.epa.gov/epawaste/conserve /tools/ warm/pdfs/Plastics.pdf (2015).

10. Ashurov N.R., Sadikov Sh.G., Khakberdiev E.O., Berdinazarov Q.N., Normurodov N.F. "Obtaining and properties of compositions based on polyethylene and gelatin", Uzbek chemical journal, 3-2020. S. $54-60$

11. Quiroz-Castillo, J. M., Rodríguez-Félix, D. E., Grijalva-Monteverde, H., del Castillo-
Castro, T., Plascencia-Jatomea, M., Rodríguez-Félix, F., \& Herrera-Franco, P. J. (2014). Preparation of extruded polyethylene/chitosan blends compatibilized with polyethylene-graftmaleic anhydride. Carbohydrate Polymers, 101, 1094-1100.

12. Bucknall, C. B. InPolymerBlends; Paul, D. R.; Bucknall, C. B., Eds.; Wiley: NewYork, 2000; Vol.2, Chapte 